\title{
Adaptive Multi-Lag for Synthetic Aperture Vector Flow Imaging
}

\author{
Villagómez Hoyos, Carlos Armando; Stuart, Matthias Bo; Jensen, Jørgen Arendt
}

\section{Published in:}

Proceedings of 2014 IEEE International Ultrasonics Symposium

Link to article, DOI:

10.1109/ULTSYM.2014.0427

Publication date:

2014

Document Version

Early version, also known as pre-print

Link back to DTU Orbit

Citation $(A P A)$ :

Villagómez Hoyos, C. A., Stuart, M. B., \& Jensen, J. A. (2014). Adaptive Multi-Lag for Synthetic Aperture Vector Flow Imaging. In Proceedings of 2014 IEEE International Ultrasonics Symposium (pp. 1722-1725). IEEE. https://doi.org/10.1109/ULTSYM.2014.0427

\section{General rights}

Copyright and moral rights for the publications made accessible in the public portal are retained by the authors and/or other copyright owners and it is a condition of accessing publications that users recognise and abide by the legal requirements associated with these rights.

- Users may download and print one copy of any publication from the public portal for the purpose of private study or research.

- You may not further distribute the material or use it for any profit-making activity or commercial gain

- You may freely distribute the URL identifying the publication in the public portal 
Paper presented at the IEEE International Ultrasonics Symposium, Chicago, Il., USA, 2014:

\section{Adaptive Multi-Lag for Synthetic Aperture Vector Flow Imaging}

Carlos A. Villagomez Hoyos, Matthias Bo Stuart and Jørgen Arendt Jensen

${ }^{1}$ Center for Fast Ultrasound Imaging,

Biomedical Engineering group, Department of Electrical Engineering, Bldg. 349,

Technical University of Denmark, DK-2800 Kgs. Lyngby, Denmark

To be published in Proceedings of IEEE International Ultrasonics Symposium, Chicago, Il., USA, 2014. 


\title{
Adaptive Multi-Lag for Synthetic Aperture Vector Flow Imaging
}

\author{
Carlos A. Villagomez Hoyos, Matthias Bo Stuart and Jørgen Arendt Jensen \\ Center for Fast Ultrasound Imaging, Department of Electrical Engineering, \\ Technical University of Denmark, DK-2800 Lyngby, Denmark
}

\begin{abstract}
The range of detectable velocities in ultrasound flow imaging is linked to the user selection of pulse repetition frequency. Whenever a region with large differences in velocity magnitude is visualized, a trade-off has to be made. This work suggests an adaptive spatio-temporaly independent, multilag method, which is performed in synthetic aperture vector flow data. Measurements are made on laminar and pulsatile, transverse flow profiles. A $7 \mathrm{MHz}$ linear array is connected to the SARUS research, and acquisitions are made on a vessel phantom with recirculating blood mimicking fluid driven by a software controlled pump. A multi-lag velocity estimation is performed, and a lag is adaptively selected for every estimation point. Results from the constant flow compared to a true parabolic profile show an improvement in relative bias from $76.99 \%$ to $0.91 \%$ and standard deviation from $13.60 \%$ to $1.83 \%$ for the low velocity flow of $0.04 \mathrm{~m} / \mathrm{s}$; and relative bias from $-2.23 \%$ to $-1.87 \%$ and standard deviation from $3.71 \%$ to $2.29 \%$ for the high velocity flow of $0.4 \mathrm{~m} / \mathrm{s}$.
\end{abstract}

\section{INTRODUCTION}

Measuring blood flow using ultrasound is a well established tool and has proven to be a valuable resource in the clinic. However, the scanner must be carefully adjusted to the range of velocities to be mapped. Whenever a region with large differences in velocity magnitudes is visualized, a trade-off has to be made to visualize either low or high velocity flow. This occurs frequently due to the pulsatile nature of blood flow, during the different stages of the cardiac cycle, or when imaging vessels of different nature such as an artery and a vein. Some scanner systems provide an automatic pulse repetition frequency (PRF) feature, which adjusts the system PRF to obtain better estimates, but they fail to provide good accuracy for both low and high velocity flow at the same time.

Advances in ultrasound imaging techniques, such as synthetic aperture technique, firstly adopted from radar 35 years ago [1], are now being used to measure blood flow such as in the work by Jensen and co-workers [2], [3], [4], [5].

More recently, Stuart et al [6] presented a high dynamic vector velocity range using synthetic aperture, but the high pulse repetition frequency required for this technique hindered the vector velocity estimation for slowly moving flow.

This is, as stated by Foster [7], due to the fact that the point of maximum correlation and the corresponding velocity estimate will vary randomly as a consequence of the random noise in the backscattered signal, if the time between echoes decreases to a small value. On the other hand, if the time between emissions is too large, more scatterers will enter and leave the investigated region during the time between echoes, and the maximum correlation of the consecutive emissions will decrease. Thus, the precision of the estimate will decrease after an increased time between echoes.

To improve the precision of the estimate, an adaptive multilag algorithm is introduced. The basic principle was presented in [8], and is based on the fact that in syntethic aperture (SA) continuous data is available, making it feasible to correlate signals for any lag at every estimation point independent of its position.

An approach suggested by Jensen [9], that merges the cross-correlation estimator with directional beamforming, is used in combination with the multi-lag approach. An adaptive algorithm is then used to select the optimal lag. The criteria used for optimization is the standard deviation of the estimates contained in an overlapping window through time.

A quick overview of the theory behind the vector velocity estimation is given in Section II-A. The multi-lag approach is introduced in Section II-B, and the details of the adaptive algorithm are given in Section II-C. The performance of the approach is assessed through measurements on parabolic laminar flows in Section III. Results obtained on a vesselmimicking phantom with an emulated pulsatile flow profile are presented.

\section{Methods}

The beamformation of the directional signals is performed as described in [10], [11]. A time domain cross-correlation is applied to obtain the true velocity magnitude for several lags; finally the adaptive algorithm selects the estimate with the lowest standard deviation.

\section{A. Vector Flow Imaging}

In $\mathrm{SA}$ vector flow imaging (VFI), lines are beamformed along the direction of the flow. The directional beamforming is based on the total transmit and receive time-of-flight, also known as a delay and sum (DAS) beamformer.

To make a fully focused line, the DAS beamformed signals $L_{i}\left(\mathbf{r}_{\mathbf{x}}\right)$ from each of the individual emission $i$ are summed to give a high resolution line

$$
g\left(\mathbf{r}_{\mathbf{x}}\right)=\sum_{i=1}^{N_{e}} L_{i}\left(\mathbf{r}_{\mathbf{x}}\right),
$$


where $N_{e}$ is the number of emissions. If the emission sequence is repeating with no delay between the last emission of one iteration of the sequence and the first emission of the next iteration, then recursive synthetic aperture (RSA) imaging is possible [12].

The directional lines $g^{t}\left(x^{\prime}, \theta_{m}\right)$ and $g^{t+\Delta t}\left(x^{\prime}, \theta_{m}\right)$ are obtained at the flow angle $\theta_{m}$ as illustrated in Fig. 1. Here the signals are $\Delta t$ seconds apart and centred at a given estimation point $\mathbf{r}_{\mathbf{x}}$. The cross-correlation approach is used to estimate the true velocity magnitude, and can be estimated by obtaining the correlation lag at the maximum of the correlation function,

$$
R_{12}\left(l, \theta_{m}\right)=\sum_{n=-N_{x c o r r} / 2}^{N_{x c o r r} / 2} g^{t}\left(n, \theta_{m}\right) g^{t+\Delta t}\left(n+l, \theta_{m}\right),
$$

where $l$ is the correlation lag and $N_{x c o r r}$ is the discrete length of the directional signals.

The probability of false peak detection is reduced by averaging over several estimates of $R_{12}$ using the assumption that the scatterers can be considered quasi-static during several pulses. A number of cross-correlations, $N_{x c}$ can thus, be averaged in the estimation.

For increased accuracy, a second-order polynomial is fit to the cross-correlation around $l_{\max }$, and the position of the maximum correlation, $\hat{l}_{\max }$, is found by the interpolation formula given in [13].

The velocity along the flow direction is then given by

$$
\hat{v}_{x^{\prime}}=\frac{\hat{l}_{\max } \cdot d_{r}}{\Delta t}
$$

where $d_{r}$ is the spatial sampling interval. The parameters used in estimating the flow are listed in Table I.

TABLE I

FLOW ESTIMATION PARAMETERS

\begin{tabular}{lll}
\hline \hline Parameter & Symbol & Value \\
\hline Spatial sampling interval & $d r$ & $0.1 \lambda$ \\
Number of samples in directional signals & $N_{\text {corr }}$ & 250 \\
Number of correlations for each estimate & $N_{x c}$ & 15 \\
Number of emissions & $N_{e}$ & 8 \\
Pulse repetition frequency & $f_{p r f}$ & $12500 \mathrm{~Hz}$ \\
\hline \hline
\end{tabular}

\section{B. Synthetic Aperture Multi-Lag}

In SA, the whole region of interest is beamformed per emission. The image beamformed from a single emission will contain higher sidelobes, therefore it is necessary to synthesize a larger aperture to regain the sidelobe level and resolution obtained with traditional techniques. This means that a sequence of emissions have to be made before a high resolution image can be obtained, as expressed in (2). Given the high system $f_{p r f}$ used in SA, several emissions $N_{e}$ can be used to synthesize a larger aperture without decreasing significantly the SNR due to the scatter motion, as investigated by Oddershede [14]; this number is normally between 3-16 emissions.

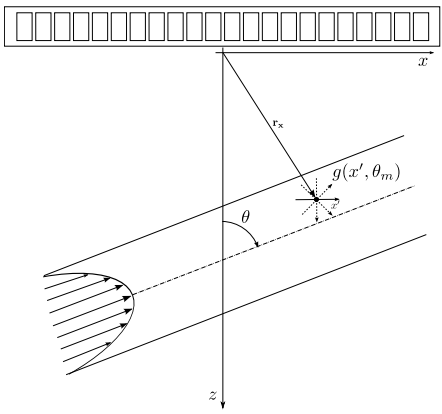

Fig. 1. Directional signals $g\left(x^{\prime}, \theta_{m}\right)$ around estimation point $\mathbf{r}_{\mathbf{x}}$.

Velocity estimation using SA require two measurements obtained in exactly the same way, so the difference is only the blood's movement between the measurements. This implies that the time between the signals that can be correlated is

$$
\Delta t=\operatorname{Lag} \cdot\left(\frac{N_{e}}{f_{p r f}}\right),
$$

where $\operatorname{Lag}=\{1,2,3, \ldots, N\}$.

Therefore, a multi-lag velocity estimate is available for each Lag value at every estimation point $\mathbf{r}_{\mathbf{x}}$ in any sampled time $t_{n}$

$$
\hat{v}_{x^{\prime}}\left(\mathbf{r}_{\mathbf{x}}, t_{n}, \text { Lag }\right),
$$

where $t_{n}=\left\{t_{0}, t_{0}+\frac{1}{f_{p r f}}, t_{0}+\frac{2}{f_{p r f}}, \ldots\right\}$, when the recursive approach is used.

\section{Adaptive Algorithm}

An optimal Lag needs to be selected to attain more accurate estimates. Since the magnitude of the velocity is not known a priori, the exactitude of the estimates cannot be used as a criteria. However, the precision of the estimates can be improved by selecting the measurement with the lowest standard deviation, and this is the criteria used for selecting the optimal Lag.

As the nature of the blood flow is pulsatile, the standard deviation cannot be estimated over the whole measurement period. Therefore, it is necessary to generate a piecewise linear approximation of the flow waveform by fitting a line to the velocity estimates. The size of the segments is selected, so that the approximation resembles the flow waveform as close as possible. The value used in this work is $2 \mathrm{~ms}$ or 25 velocity estimates.

The optimal Lag is selected by minimizing the standard deviation of subtracting a piecewise linear approximation from the velocity estimate at each point as denoted by

$$
\operatorname{Lag}\left(\mathbf{r}_{\mathbf{x}}, t n\right)=\arg \min _{\operatorname{Lag}}\left[\operatorname{std}\left\{\hat{v^{\prime}}\left(\mathbf{r}_{\mathbf{x}}, t_{n}, \operatorname{Lag}\right)-\hat{v}_{p l}\left(\mathbf{r}_{\mathbf{x}}, t_{n}, L a g\right)\right\}\right],
$$

where $\hat{v}^{\prime}\left(\mathbf{r}_{\mathbf{x}}, t_{n}\right.$, Lag $)$ is the segment from the estimated velocity and $\hat{v}_{p l}\left(\mathbf{r}_{\mathbf{x}}, t_{n}, L a g\right)$ is the piecewise linear approximation at that segment.

Finally the $\operatorname{Lag}\left(\mathbf{r}_{\mathbf{x}}, t n\right)$ is smoothed using a Gaussian filter to reduce Lag jumps due to noise. For this work the size of the window used was $49 \times 49(4.9[\mathrm{~mm}]$ x $3.9[\mathrm{~ms}])$. 


\section{MEASUREMENT RESUlTS}

Data for the velocity estimation approach is recorded using the SARUS experimental scanner [15]. A $7 \mathrm{MHz}$ linear array transducer, type 8670 (BK Medical, Herlev, Denmark), is employed, and velocity estimates are obtained by beamforming directionally at an estimation point and cross-correlating the two measurements. Eight emissions are used in the sequence and the virtual sources are equally distributed around the center of the aperture. The virtual sources are located $40 \mathrm{~mm}$ behind the transducer $(F \#=-2)$. The system $f_{\text {prf }}$ is set at $12.5 \mathrm{kHz}$. A 1-cycle sinusoidal pulse with 64 elements are used in transmit, while all 128 elements are used in receive. All 128 channels are sampled at a frequency of $35 \mathrm{MHz}$.

A flow vessel phantom with a beam-to-flow angle of 90 degrees is used. The vessel has an internal diameter of $7 \mathrm{~mm}$ at a depth of $18 \mathrm{~mm}$. For the measurements, a Shelley Medical software controlled pump (Toronto, Canada) is used to circulate blood-mimicking fluid[16] (Danish Phantom Design, Frederikssund, Denmark) in a closed loop circuit. The pump was used to imitate two different flow profiles: constant flow and a carotid flow.
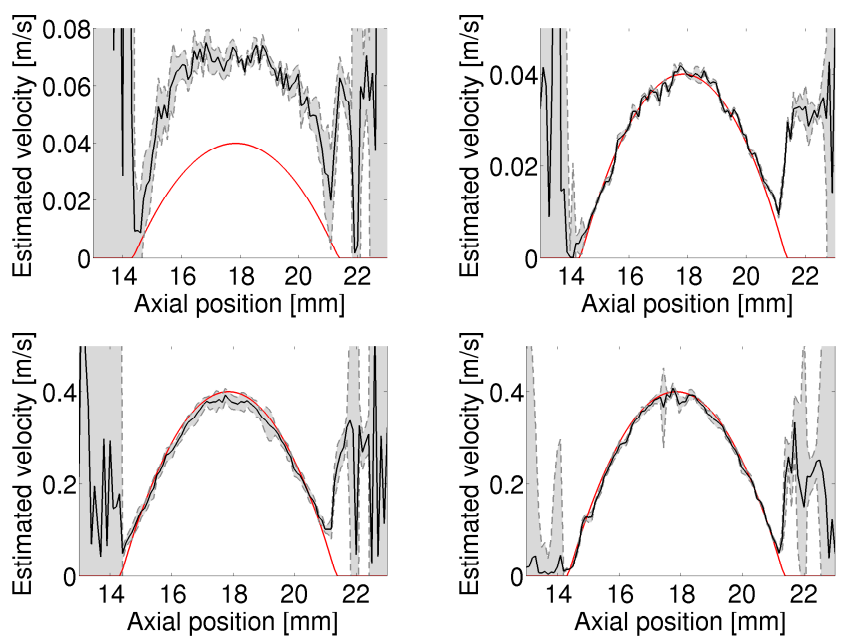

Fig. 2. Velocity profiles obtained at a beam-to-flow angle of 90 degrees with true parabolic profile in red. (Top) Peak velocity of $0.04 \mathrm{~m} / \mathrm{s}$; (Left) without adaptive multi-lag; and (Right) with adaptive multi-lag . (Bottom) Peak velocity of $0.4 \mathrm{~m} / \mathrm{s}$; (Left) without adaptive multi-lag; and (Right) with adaptive multi-lag.

\section{A. Parabolic Constant Flow}

The velocities are estimated with the adaptive multi-lag method and compared to the fixed lag approach. Data are acquired for $80 \mathrm{~ms}$, yielding 1000 profiles from which the mean, standard deviation, and bias are calculated relative to a parabolic true profile. Two distinct flow rates are used for assessing the performance in both high and low velocity flow; the flow rate is set to $10 \mathrm{ml} / \mathrm{sec}$ corresponding to an estimated peak velocity of $0.4 \mathrm{~m} / \mathrm{s}$ for the high velocity flow, and a flow rate of $1 \mathrm{ml} / \mathrm{sec}$ corresponding to an estimated peak velocity of $0.04 \mathrm{~m} / \mathrm{s}$ for the low velocity flow.

A side-by-side comparison between the flow profiles is shown in Fig. 2. On top, the low velocity flows are shown for the traditional approach (left) and the proposed algorithm (right). The relative standard deviation and bias are $13.60 \%$ and $76.99 \%$, for the traditional approach and $1.83 \%$ and $0.91 \%$, for the proposed algorithm respectively.

On the bottom, the high velocity flows are shown. The relative standard deviation and bias are $3.71 \%$ and $-2.23 \%$, for the traditional approach and $2.29 \%$ and $-1.87 \%$, for the proposed algorithm respectively.

\section{B. Pulsatile flow}

A pulsatile carotid flow is programmed in the pump. The peak flow rate is set to $25 \mathrm{ml} / \mathrm{sec}$ and the period between pulses is set to 0.820 seconds. Three seconds of data are acquire yielding around 37000 estimates. A multi-lag velocity estimation is performed and a lag is adaptively selected at every estimation point, and are shown as the $X Y$ plane in Fig. 3 . The velocity estimates are shown as a surface, where the $X$ axis is the spatial dimension and the center line, and the $Y$ axis is the temporal dimension showing the progression of the pulse through time. In the lower part of Fig. 3 a fixed lag is used, represented by a solid color on the $X Y$ plane, and the velocity estimates are also shown as a surface. The estimates in this figure are more noisy compared to the multilag approach. The lower velocities are specially affected when using a lower lag, since the backscattered noise degrades the signal. In contrast the $X Y$ plane in upper part of Fig. 3, which represent the used lag, follows correctly the shape of the pulse; selecting a lower lag around the peak flow in the center of the vessel and increasing it towards the outer part of the vessel. Since the velocity range of adjacent lags overlap each other a more uniform lag selection map is not needed.

\section{CONCLUSION}

This paper demonstrates that with SA flow imaging it is possible to measure both low and high velocities with good accuracy and a high frame rate. The results show that there is a substantial improvement in the low velocity flow, and by using a different lag not only the standard deviation is lowered but also the bias in the estimates. This paper demonstrates that availability of continuous data that SA provides is a great asset for obtaining better velocity estimates in a wider range of velocities without loss of spatial resolution.

\section{ACKNOWLEDGEMENT}

This work was supported by grant 82-2012-4 from the Danish Advanced Technology Foundation and by BK Medical Aps.

\section{REFERENCES}

[1] C. B. Burckhardt, P.-A. Grandchamp, and H. Hoffmann, "An experimental $2 \mathrm{MHz}$ synthetic aperture sonar system intended for medical use," IEEE Trans. Son. Ultrason., vol. 21, no. 1, pp. 1-6, January 1974.

[2] S. I. Nikolov and J. A. Jensen, "Velocity estimation using synthetic aperture imaging," in Proc. IEEE Ultrason. Symp., 2001, pp. 1409-1412.

[3] J. A. Jensen and S. I. Nikolov, "Transverse flow imaging using synthetic aperture directional beamforming," in Proc. IEEE Ultrason. Symp., 2002, pp. 1488-1492. 

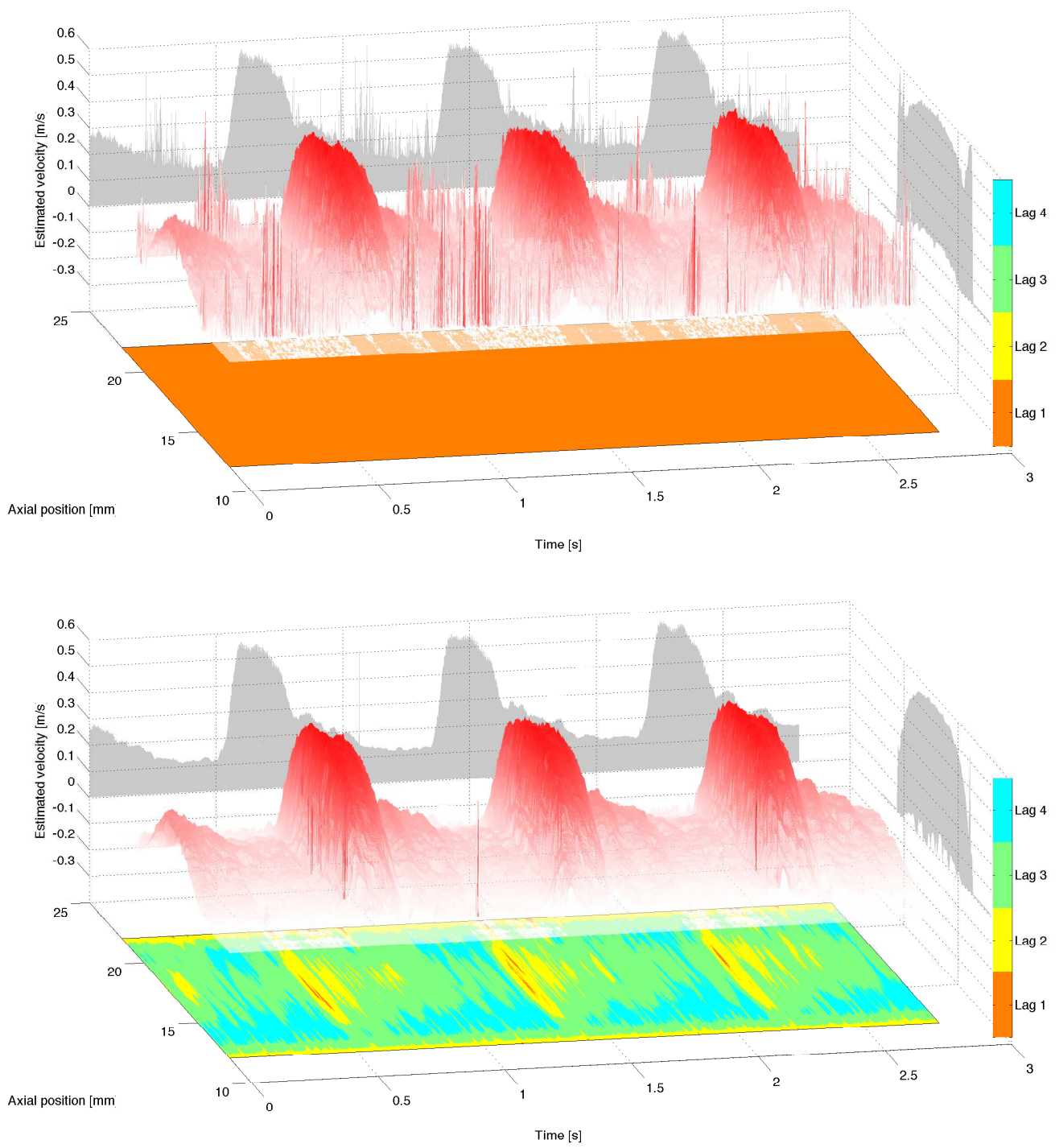

Fig. 3. Velocity profiles of the center line of the vessel through time. Projections of the two axis are shown as shadows. (Top) The velocity estimates using a fixed lag. (Bottom) The velocity estimates using the adaptive algorithm.

[4] S. I. Nikolov and J. A. Jensen, "In-vivo Synthetic Aperture Flow Imaging in Medical Ultrasound," IEEE Trans. Ultrason., Ferroelec., Freq. Contr., vol. 50, no. 7, pp. 848-856, 2003.

[5] J. A. Jensen and S. I. Nikolov, "A method for real-time three-dimensional vector velocity imaging," in Proc. IEEE Ultrason. Symp., 2003, pp. $1582-1585$.

[6] M. B. Stuart, B. G. Tomov, M. J. Pihl, and J. A. Jensen, "High frame rate synthetic aperture duplex imaging," in Proc. IEEE Ultrason. Symp., July 2013, pp. 623-626.

[7] S. G. Foster, P. M. Embree, and W. D. O’Brien, "Flow velocity profile via time-domain correlation: Error analysis and computer simulation," IEEE Trans. Ultrason., Ferroelec., Freq. Contr., vol. 37, pp. 164-175, 1990.

[8] C. Villagomez-Hoyos, M. B. Stuart, and J. A. Jensen, "Increasing the dynamic range of synthetic aperture vector flow imaging," in Proc. SPIE Med. Imag., vol. 9040, 2014, p. In press.

[9] J. A. Jensen, "Velocity vector estimation in synthetic aperture flow and B-mode imaging," in IEEE International Symposium on Biomedical imaging from nano to macro, 2004, pp. 32-35.

[10] — - "Directional velocity estimation using focusing along the flow direction: I: Theory and simulation," IEEE Trans. Ultrason., Ferroelec., Freq. Contr., vol. 50, pp. 857-872, 2003.
[11] J. A. Jensen and R. Bjerngaard, "Directional velocity estimation using focusing along the flow direction: II: Experimental investigation," IEEE Trans. Ultrason., Ferroelec., Freq. Contr., vol. 50, pp. 873-880, 2003.

[12] S. I. Nikolov, K. Gammelmark, and J. A. Jensen, "Recursive ultrasound imaging," in Proc. IEEE Ultrason. Symp., vol. 2, 1999, pp. 1621-1625.

[13] S. G. Foster, "A pulsed ultrasonic flowmeter employing time domain methods," Ph.D. dissertation, Dept. Elec. Eng., University of Illinois, Urbana, Ill., 1985.

[14] N. Oddershede and J. A. Jensen, "Effects influencing focusing in synthetic aperture vector flow imaging," IEEE Trans. Ultrason., Ferroelec., Freq. Contr, vol. 54, no. 9, pp. 1811-1825, 2007.

[15] J. A. Jensen, H. Holten-Lund, R. T. Nilsson, M. Hansen, U. D. Larsen, R. P. Domsten, B. G. Tomov, M. B. Stuart, S. I. Nikolov, M. J. Pihl, Y. Du, J. H. Rasmussen, and M. F. Rasmussen, "SARUS: A synthetic aperture real-time ultrasound system," IEEE Trans. Ultrason., Ferroelec., Freq. Contr., vol. 60, no. 9, pp. 1838-1852, 2013.

[16] K. V. Ramnarine, D. K. Nassiri, P. R. Hoskins, and J. Lubbers, "Validation of a new blood mimicking fluid for use in Doppler flow test objects," Ultrasound Med. Biol., vol. 24, pp. 451-459, 1998. 